\title{
Joint 2D-DOD and 2D-DOA Estimation in Bistatic MIMO Radar
}

\author{
Hao Liang, Chen Cui, Jian Yu \\ 401 Laboratory, Department of Communication Countermeasure, \\ Electronic Engineering Institute, Hefei 230037, China
}

Keywords: Unitary Transformation; Angle Estimation; Bistatic MIMO Radar; Cross Array

\begin{abstract}
In this paper, the issue of two-dimensional (2D) direction of arrival (DOA) and twodimensional (2D) direction of departure (DOD) estimation for bistatic multiple-input-multipleoutput (MIMO) radar with cross array is investigated. A novel multidimensional angle estimation methods based on unitary transformation is proposed. Firstly, a new unitary matrix is designed using the central symmetry of cross array at transmit and receive site. Then both the rotational invariance relationships in transmit and receive array can be turned into the real-value field via the unitary transformation. And 2D-DOA and 2D-DOD joint estimations can be obtained. The proposed method, without costing the aperture of array and peak searching, can realize multidimensional angle estimation with automatic pairing and provides better estimation performance than ESPRIT. Compared with the traditional unitary transformation, the steering vectors of transmit and receive array can be turned into a real-value field via the unitary matrix and transformation in our scheme, respectively, Therefore, the proposed method can realize that the eigen-value decomposition and parameter estimation can be computed absolutely in a real-value field, with a result of a lower computation complexity. Simulation results verify the correctness of theoretical analysis and the effectiveness of proposed algorithm.
\end{abstract}

\section{Introduction}

Multiple-input-multiple-output (MIMO)radar, which utilizes multiple antennas to simultaneously transmit orthogonal waveforms and uses multiple antennas to receive the reflected signals, has many potential advantages over conventional phased-array radars ${ }^{[1-4]}$. According to the antennas configuration, MIMO radars can be classified into two types: One is distributed MIMO radar whose transmit and receive arrays antennas are widely spaced to obtain coherent processing gain for solving target scintillation problem. Another one is collocated MIMO radar (including bistatic and monostatic ones), which is closely spaced in both transmit array and receive array to achieve unambiguous angle estimation ${ }^{[1,3]}$.

The direction of arrival (DOA) estimation algorithms in collocated MIMO radar have attracted a lot of attention and have been investigated by many researchers. In order to achieve the effective estimated parameters, traditional high-resolution direction finding (DF) algorithms, such as the maximum likelihood (ML) estimate, multiple signal classification (MUSIC) estimate, estimation of signal parameters by rotational invariance techniques (ESPRIT) and so on, have been widely used in the target' parameter estimation of MIMO radar ${ }^{[5]-[8]}$ and have reached superior performance compared with the conventional methods. In these DF algorithms, Conventional ML, Capon and MUSIC algorithms have been used for angle estimation regardless of the array manifold in MIMO radar, but they need spatial spectrum peak searching with high computation cost. Estimation of signal parameters via rotational invariance techniques (ESPRIT) in [7] utilizes the rotational invariance in the subspace to obtain automatically paired estimations of the angles, and an ESPRITbased method can be used for joint angle and Doppler frequency estimation ${ }^{\text {[9] }}$. Unitary ESPRIT [8], which was proposed for two-dimensional source location in array signal processing [10], was employed to transform the complex-valued computation into a real-valued one. Compared to ESPRIT, a slight improvement of the angle estimation performance can be achieved via unitary ESPRIT due to the increasing of virtual sample number.

In this paper, the issue of two-dimensional (2D) direction of arrival (DOA) and 2D direction of departure (DOD) estimation for bistatic multiple-input-multiple-output (MIMO) radar with cross 
array is investigated. A novel multidimensional angle estimation methods based on unitary transformation is proposed. The proposed method, without costing the aperture of array and peak searching, can realize multidimensional angle estimation with automatic pairing and provides better estimation performance than ESPRIT. The steering vectors of transmit and receive array can be turned into a real-value field via the unitary matrix and transformation in our scheme, respectively, the proposed method can realize that the eigen-value decomposition and parameter estimation can be computed absolutely in a real-value field, with a result of a lower computation complexity.

\section{Signal model}

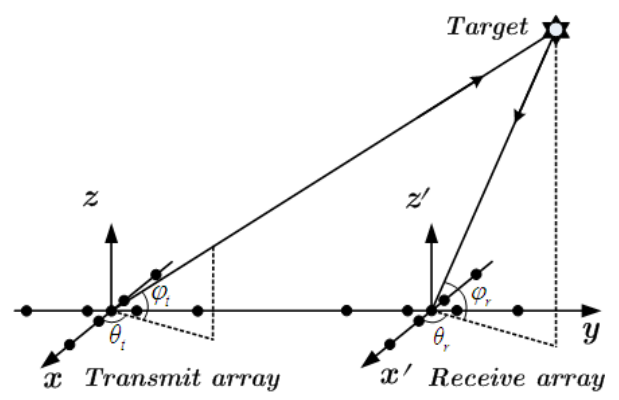

Figure. 1 bistatic MIMO radar with cross array

Consider a bistatic MIMO radar system equipped with the same cross array for the transmit and receive sites in which the cross array consist of two symmetric uniform linear arrays (ULA) arranged in xoy plane with $2 M_{x}-1$ elements on $x$ axis and $2 M_{y}-1$ elements on $y$ axis, respectively and coinciding with the center of the cross array (it is denoted as the origin). The arrays of the transmitter and receiver have been collocated with an inter element distance of a half-wavelength. And transmit and receive arrays antenna structure is shown in Fig.1. Assume that there are $K$ targets with different doppler frequencies assumed to be located at the same range bin. And the output of the matched filters at the receiver can be expressed as

$$
\boldsymbol{x}(t)=\left[\boldsymbol{a}_{r}\left(\vartheta_{r 1}, \phi_{r 1}\right) \otimes \boldsymbol{a}_{t}\left(\vartheta_{t 1}, \phi_{t 1}\right), \cdots, \boldsymbol{a}_{r}\left(\vartheta_{r K}, \phi_{r K}\right) \otimes \boldsymbol{a}_{t}\left(\vartheta_{t K}, \phi_{t K}\right)\right] \boldsymbol{\beta}(t)+\boldsymbol{n}(t)
$$

Where $\vartheta_{r}, \phi_{r}$ are the elevation and azimuth of the 2D-DOA of the $k$ th target with respect to the receive array normal; $\vartheta_{t}, \phi_{t}$ are the elevation and azimuth of the 2D-DOD of the $k$ th target with respect to the transmit array normal. $\boldsymbol{\beta}(t)=\left[\beta_{1}(t), \cdots, \beta_{K}(t)\right]^{T}, \beta_{k}(t)=\alpha_{k} \exp \left(j 2 \pi f_{k} t\right)$ with $f_{k}$ being Doppler frequency and $\alpha_{k}$ the amplitude. $n(t)$ is an Gaussian white noise vector of zeros mean and covariance matrix $\sigma_{n}^{2} \boldsymbol{I}_{M_{t} M_{r}}$.Define $\boldsymbol{G}=\left[\boldsymbol{g}\left(\vartheta_{t 1}, \phi_{t 1} ; \vartheta_{r 1}, \phi_{r 1}\right), \cdots, \boldsymbol{g}\left(\vartheta_{t K}, \phi_{t K} ; \vartheta_{r K}, \phi_{r K}\right)\right]$ and $\boldsymbol{g}\left(\vartheta_{t}, \phi_{t} ; \vartheta_{r}, \phi_{r}\right)$ $=\boldsymbol{a}_{r}\left(\vartheta_{r}, \phi_{r}\right) \otimes \boldsymbol{a}_{t}\left(\vartheta_{t}, \phi_{t}\right)$. The receive and transmit steering vectors can be expressed as

$$
\begin{aligned}
& \boldsymbol{a}_{t}\left(\vartheta_{t}, \phi_{t}\right)=\left[\overline{\boldsymbol{a}}_{t y}^{+}\left(\vartheta_{t}, \phi_{t}\right)^{T}, \boldsymbol{a}_{t x}\left(\vartheta_{t}, \phi_{t}\right)^{T}, \underline{\boldsymbol{a}}_{t y}^{-}\left(\vartheta_{t}, \phi_{t}\right)^{T}\right]^{T} \\
& \boldsymbol{a}_{r}\left(\vartheta_{r}, \phi_{r}\right)=\left[\overline{\boldsymbol{a}}_{r y}^{+}\left(\vartheta_{r}, \phi_{r}\right)^{T}, \boldsymbol{a}_{r x}\left(\vartheta_{r}, \phi_{r}\right)^{T}, \underline{\boldsymbol{a}}_{r y}^{-}\left(\vartheta_{r}, \phi_{r}\right)^{T}\right]^{T}
\end{aligned}
$$

Where $\boldsymbol{a}_{t x}\left(\vartheta_{t}, \phi_{t}\right)=\left[\boldsymbol{a}_{t x}^{+}\left(\vartheta_{t}, \phi_{t}\right)^{T}, \underline{a}_{t x}^{-}\left(\vartheta_{t}, \phi_{t}\right)^{T}\right]^{T}=\left[\overline{\boldsymbol{a}}_{t x}^{+}\left(\vartheta_{t}, \phi_{t}\right)^{T}, \boldsymbol{a}_{t x}^{-}\left(\vartheta_{t}, \phi_{t}\right)^{T}\right]^{T}$, $\boldsymbol{a}_{r x}\left(\vartheta_{r}, \phi_{r}\right)=\left[\boldsymbol{a}_{r x}^{+}\left(\vartheta_{r}, \phi_{r}\right)^{T}, \underline{\boldsymbol{a}}_{r x}^{-}\left(\vartheta_{r}, \phi_{r}\right)^{T}\right]^{T}=\left[\overline{\boldsymbol{a}}_{r x}^{+}\left(\vartheta_{r}, \phi_{r}\right)^{T}, \boldsymbol{a}_{r x}^{-}\left(\vartheta_{r}, \phi_{r}\right)^{T}\right]^{T}$. Here $\overline{\boldsymbol{e}}$ and $\underline{\boldsymbol{e}}$ denote the first and last length $(\boldsymbol{e})-1$ rows of vector $\boldsymbol{e}$. And

$$
\begin{array}{ll}
\boldsymbol{a}_{t y}^{+}\left(\vartheta_{t}, \phi_{t}\right)=\left[\exp \left(j \kappa_{M_{t y}-1}^{t y}\right), \cdots, \exp \left(j \kappa_{0}^{t y}\right)\right]^{T} & \boldsymbol{a}_{t y}^{-}\left(\vartheta_{t}, \phi_{t}\right)=\left[\exp \left(j \kappa_{0}^{t y}\right), \cdots, \exp \left(j \kappa_{-\left(M_{t y}-1\right)}^{t y}\right)\right]^{T} \\
\boldsymbol{a}_{t x}^{+}\left(\vartheta_{t}, \phi_{t}\right)=\left[\exp \left(j \kappa_{M_{t x}-1}^{t x}\right), \cdots, \exp \left(j \kappa_{0}^{t x}\right)\right]^{T} & \boldsymbol{a}_{t x}^{-}\left(\vartheta_{t}, \phi_{t}\right)=\left[\exp \left(j \kappa_{0}^{t x}\right), \cdots, \exp \left(j \kappa_{-\left(M_{t x}-1\right)}^{t x}\right)\right]^{T}
\end{array}
$$




$$
\begin{array}{ll}
\boldsymbol{a}_{r y}^{+}\left(\vartheta_{r}, \phi_{r}\right)=\left[\exp \left(j \kappa_{M_{r y}-1}^{r y}\right), \cdots, \exp \left(j \kappa_{0}^{r y}\right)\right]^{T} & \boldsymbol{a}_{r y}^{-}\left(\vartheta_{r}, \phi_{r}\right)=\left[\exp \left(j \kappa_{0}^{r y}\right), \cdots, \exp \left(j \kappa_{-\left(M_{r y}-1\right)}^{r y}\right)\right]^{T} \\
\boldsymbol{a}_{r x}^{+}\left(\vartheta_{r}, \phi_{r}\right)=\left[\exp \left(j \kappa_{M_{r x}-1}^{r x}\right), \cdots, \exp \left(j \kappa_{0}^{r x}\right)\right]^{T} & \boldsymbol{a}_{r x}^{-}\left(\vartheta_{r}, \phi_{r}\right)=\left[\exp \left(j \kappa_{0}^{r x}\right), \cdots, \exp \left(j \kappa_{-\left(M_{r x}-1\right)}^{r x}\right)\right]^{T}
\end{array}
$$

Where $\kappa_{m_{t y}}^{t y}=-\pi m_{t y} \sin \vartheta_{t} \cos \phi_{t}, \kappa_{m_{t x}}^{t x}=-\pi m_{t x} \cos \vartheta_{t} \cos \phi_{t}, \kappa_{m_{r y}}^{r y}=-\pi m_{r y} \sin \vartheta_{r} \cos \phi_{r}, \kappa_{m_{r x}}^{r x}=-\pi m_{r x} \cos \vartheta_{r} \cos \phi_{r}$; $m_{t y} \in\left[-\left(M_{t y}-1\right), M_{t y}-1\right], \quad m_{t x} \in\left[-\left(M_{t x}-1\right), M_{t x}-1\right], \quad m_{r y} \in\left[-\left(M_{r y}-1\right), M_{r y}-1\right], \quad m_{r x} \in\left[-\left(M_{r x}-1\right), M_{r x}-1\right]$.

\section{Joint DOD and DOA Estimation}

According to the signal model, the transmit and receive steering vectors can be calculated as

$$
\begin{aligned}
& \boldsymbol{Q}_{M_{t}}^{H} \boldsymbol{a}_{t}\left(\vartheta_{t}, \phi_{t}\right)=\boldsymbol{h}_{t}\left(\vartheta_{t}, \phi_{t}\right)=\left[\overline{\boldsymbol{h}}_{t y}^{+}\left(\vartheta_{t}, \phi_{t}\right)^{T}, \overline{\boldsymbol{h}}_{t x}^{+}\left(\vartheta_{t}, \phi_{t}\right)^{T}, 1, \overline{\boldsymbol{h}}_{t x}^{-}\left(\vartheta_{t}, \phi_{t}\right)^{T}, \overline{\boldsymbol{h}}_{t y}^{-}\left(\vartheta_{t}, \phi_{t}\right)^{T}\right]^{T} \\
& \boldsymbol{Q}_{M_{r}}^{H} \boldsymbol{a}_{r}\left(\vartheta_{r}, \phi_{r}\right)=\boldsymbol{h}_{r}\left(\vartheta_{r}, \phi_{r}\right)=\left[\overline{\boldsymbol{h}}_{r y}^{+}\left(\vartheta_{r}, \phi_{r}\right)^{T}, \overline{\boldsymbol{h}}_{r x}^{+}\left(\vartheta_{r}, \phi_{r}\right)^{T}, 1, \overline{\boldsymbol{h}}_{r x}^{-}\left(\vartheta_{r}, \phi_{r}\right)^{T}, \overline{\boldsymbol{h}}_{r y}^{-}\left(\vartheta_{r}, \phi_{r}\right)^{T}\right]^{T}
\end{aligned}
$$

Here unitary matrix $\boldsymbol{Q}_{M_{t}}$ and $\boldsymbol{Q}_{M_{r}}$ are the same as the expression in [8]. And

$$
\begin{aligned}
& \boldsymbol{h}_{t y}^{+}\left(\vartheta_{t}, \phi_{t}\right)=\sqrt{2}\left[\cos \left(\boldsymbol{\kappa}_{M_{t y}-1}^{t y}\right), \cdots, \cos \left(\boldsymbol{\kappa}_{0}^{t y}\right)\right]^{T}, \boldsymbol{h}_{t y}^{-}\left(\vartheta_{t}, \phi_{t}\right)=\sqrt{2}\left[-\sin \left(\boldsymbol{\kappa}_{M_{t y}-1}^{t y}\right), \cdots,-\sin \left(\boldsymbol{\kappa}_{0}^{t y}\right)\right]^{T} \\
& \boldsymbol{h}_{t x}^{+}\left(\vartheta_{t}, \phi_{t}\right)=\sqrt{2}\left[\cos \left(\boldsymbol{\kappa}_{M_{t x}-1}^{t x}\right), \cdots, \cos \left(\boldsymbol{\kappa}_{0}^{t x}\right)\right]^{T}, \boldsymbol{h}_{t x}^{-}\left(\vartheta_{t}, \phi_{t}\right)=\sqrt{2}\left[-\sin \left(\boldsymbol{\kappa}_{M_{t x}-1}^{t x}\right), \cdots,-\sin \left(\boldsymbol{\kappa}_{0}^{t x}\right)\right]^{T} \\
& \boldsymbol{h}_{r y}^{+}\left(\vartheta_{r}, \phi_{r}\right)=\sqrt{2}\left[\cos \left(\boldsymbol{\kappa}_{M_{r y}-1}^{r y}\right), \cdots, \cos \left(\kappa_{0}^{r y}\right)\right]^{T}, \boldsymbol{h}_{r y}^{-}\left(\vartheta_{r}, \phi_{r}\right)=\sqrt{2}\left[-\sin \left(\boldsymbol{\kappa}_{M_{r y}-1}^{r y}\right), \cdots,-\sin \left(\boldsymbol{\kappa}_{0}^{r y}\right)\right]^{T} \\
& \boldsymbol{h}_{r x}^{+}\left(\vartheta_{r}, \phi_{r}\right)=\sqrt{2}\left[\cos \left(\boldsymbol{\kappa}_{M_{r x}-1}^{r x}\right), \cdots, \cos \left(\boldsymbol{\kappa}_{0}^{r x}\right)\right]^{T}, \boldsymbol{h}_{r x}^{-}\left(\vartheta_{r}, \phi_{r}\right)=\sqrt{2}\left[-\sin \left(\boldsymbol{\kappa}_{M_{r x}-1}^{r x}\right), \cdots,-\sin \left(\boldsymbol{\kappa}_{0}^{r x}\right)\right]^{T}
\end{aligned}
$$

According to the equation (4), transmit and receive steering vector can be transformed into a real valued vector, respectively. Define $\tilde{Q}_{M_{t} M_{r}}=Q_{M_{r}} \otimes Q_{M_{t}}$, and the steering vector can be transformed by

$$
\tilde{\boldsymbol{Q}}_{M_{t} M_{r}}^{H} \boldsymbol{g}\left(\vartheta_{t}, \phi_{t} ; \vartheta_{r}, \phi_{r}\right)=\left[\boldsymbol{Q}_{M_{r}}^{H} \boldsymbol{a}_{r}\left(\vartheta_{r}, \phi_{r}\right)\right] \otimes\left[\boldsymbol{Q}_{M_{t}}^{H} \boldsymbol{a}_{t}\left(\vartheta_{t}, \phi_{t}\right)\right]=\boldsymbol{h}_{r}\left(\vartheta_{r}, \phi_{r}\right) \otimes \boldsymbol{h}_{t}\left(\vartheta_{t}, \phi_{t}\right)=\boldsymbol{h}\left(\vartheta_{t}, \phi_{t} ; \vartheta_{r}, \phi_{r}\right)
$$

Therefore, using the unitary matrix $\tilde{\boldsymbol{Q}}_{M_{t} M_{r}}$, the covariance matrix of received data can be transformed into a real-field. Before the unitary transformation, the center-hermitian covariance matrix (CHM) via the forward /backward (FB) averaging in $[8,10]$ can be achieved by

$$
\boldsymbol{R}_{f}=\left(\boldsymbol{R}_{x}+\boldsymbol{\Pi}_{M_{t} M_{r}} \boldsymbol{R}_{x}^{*} \boldsymbol{\Pi}_{M_{t} M_{r}}\right) / 2=\boldsymbol{G}\left(\boldsymbol{R}_{\boldsymbol{\beta}}+\boldsymbol{R}_{\beta}^{*}\right) \boldsymbol{G}^{H} / 2+\sigma_{n}^{2} \boldsymbol{I}_{M_{t} M_{r}}
$$

Where $\boldsymbol{R}_{x}=E\left\{\boldsymbol{x}(t) \boldsymbol{x}^{H}(t)\right\}=\boldsymbol{G R}_{\beta} \boldsymbol{G}^{H}+\sigma_{n}^{2} \boldsymbol{I}_{M_{t} M_{r}}$ denotes the corresponding CM in complex field. $\Pi_{M_{t} M_{r}}$ is an exchange matrix with $M_{t} M_{r}$ ones on its anti-diagonal and zeros elsewhere, And $\boldsymbol{R}_{\boldsymbol{\beta}}=E\left\{\boldsymbol{\beta}(t) \boldsymbol{\beta}(t)^{H}\right\}, \boldsymbol{\Pi}_{M_{t} M_{r}} \boldsymbol{G}^{*}=\boldsymbol{G}, \boldsymbol{G}^{T} \boldsymbol{\Pi}_{M_{t} M_{r}}=\boldsymbol{G}^{H}$. If we perform a unitary transformation on the covariance matrix in (7), then

$$
\boldsymbol{R}_{C M}=\tilde{\boldsymbol{Q}}_{M_{t} M_{r}}^{H} \boldsymbol{R}_{f} \tilde{\boldsymbol{Q}}_{M_{t} M_{r}}=\boldsymbol{H} \operatorname{Re}\left(\boldsymbol{R}_{\beta}\right) \boldsymbol{H}^{H}+\sigma_{n}^{2} \boldsymbol{I}_{M_{t} M_{r}}
$$

Where $\boldsymbol{H}=\left[\boldsymbol{h}\left(\vartheta_{t 1}, \phi_{t 1} ; \vartheta_{r 1}, \phi_{r 1}\right), \cdots, \boldsymbol{h}\left(\vartheta_{t K}, \phi_{t K} ; \vartheta_{r K}, \phi_{r K}\right)\right]$; obviously, the real-valued covariance matrix $\boldsymbol{R}_{C M}$ can be obtained by the unitary transformation for $\boldsymbol{R}_{f}$. Through the eigen-decomposition of $\boldsymbol{R}_{C M}$, the corresponding real-valued signal subspace $\boldsymbol{U}_{S}$ can be obtained. According to ${ }^{[4]}$, the signal subspace $\boldsymbol{U}_{\boldsymbol{S}}$ is related to $\boldsymbol{H}$ as $\boldsymbol{U}_{\boldsymbol{S}}=\boldsymbol{H} \boldsymbol{T}=\tilde{\boldsymbol{Q}}_{M_{t} M_{r}}^{H} \boldsymbol{G T}$, where $\boldsymbol{T}$ is a nonsingular matrix. According to (2), the transmit and receive steering vector satisfy

$$
\begin{aligned}
& \exp \left(j \kappa_{-1}^{t y}\right) \boldsymbol{J}_{t}^{y 1} \boldsymbol{J}_{t y} \boldsymbol{a}_{t}\left(\vartheta_{t}, \phi_{t}\right)=\boldsymbol{J}_{t}^{y 2} \boldsymbol{J}_{t y} \boldsymbol{a}_{t}\left(\vartheta_{t}, \phi_{t}\right) \exp \left(j \kappa_{-1}^{r y}\right) \boldsymbol{J}_{r}^{y 1} \boldsymbol{J}_{r y} \boldsymbol{a}_{r}\left(\vartheta_{r}, \phi_{r}\right)=\boldsymbol{J}_{r}^{y 2} \boldsymbol{J}_{r y} \boldsymbol{a}_{r}\left(\vartheta_{r}, \phi_{r}\right) \\
& \exp \left(j \kappa_{-1}^{t x}\right) \boldsymbol{J}_{t}^{x 1} \boldsymbol{J}_{t x} \boldsymbol{a}_{t}\left(\vartheta_{t}, \phi_{t}\right)=\boldsymbol{J}_{t}^{x 2} \boldsymbol{J}_{t x} \boldsymbol{a}_{t}\left(\vartheta_{t}, \phi_{t}\right) \exp \left(j \kappa_{-1}^{r x}\right) \boldsymbol{J}_{r}^{\chi 1} \boldsymbol{J}_{r x} \boldsymbol{a}_{r}\left(\vartheta_{r}, \phi_{r}\right)=\boldsymbol{J}_{r}^{\chi 2} \boldsymbol{J}_{r x} \boldsymbol{a}_{r}\left(\vartheta_{r}, \phi_{r}\right)
\end{aligned}
$$




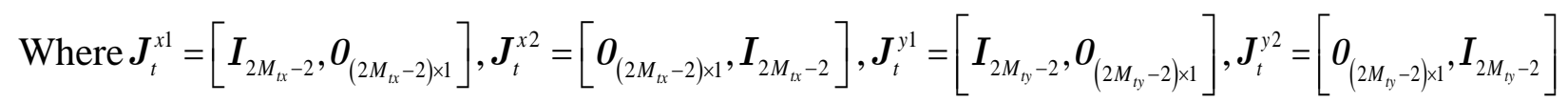

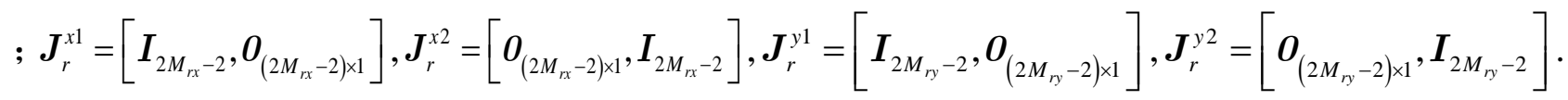
And $\boldsymbol{J}_{t y}, \boldsymbol{J}_{t x}, \boldsymbol{J}_{r x}, \boldsymbol{J}_{r y}$ can be calculated as $\boldsymbol{J}_{r x}=\left[\begin{array}{lll}\boldsymbol{O}_{M_{r y}-1} & \boldsymbol{I}_{2 M_{r x}-1} & \boldsymbol{O}_{M_{r y}-1}\end{array}\right], \boldsymbol{J}_{t x}=\left[\begin{array}{lll}\boldsymbol{O}_{M_{y y}-1} & \boldsymbol{I}_{2 M_{x}-1} & \boldsymbol{O}_{M_{\mathrm{yy}}-1}\end{array}\right]$,

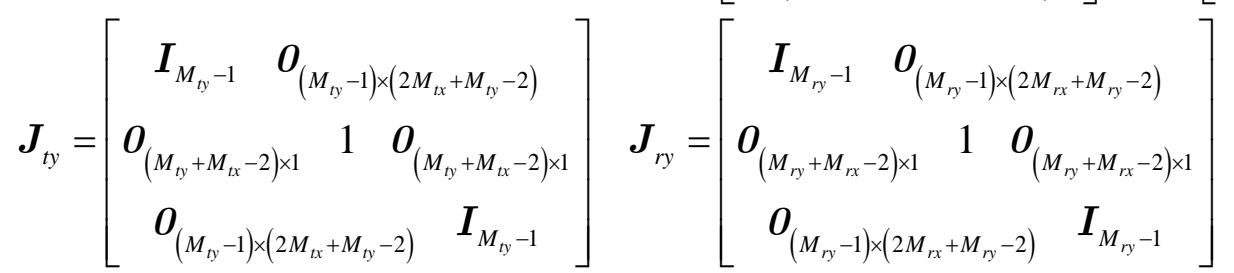

According to (6), we can get

$$
\begin{aligned}
& \tan \left(\boldsymbol{\kappa}_{-1}^{\mathrm{ty}} / 2\right)\left(\boldsymbol{K}_{t}^{\mathrm{y} 1} \boldsymbol{T}_{t y} \otimes \boldsymbol{I}_{M_{r}}\right) \boldsymbol{h}\left(\vartheta_{t}, \phi_{t} ; \vartheta_{r}, \phi_{r}\right)=\left(\boldsymbol{K}_{t}^{y 2} \boldsymbol{T}_{t y} \otimes \boldsymbol{I}_{M_{r}}\right) \boldsymbol{h}\left(\vartheta_{t}, \phi_{t} ; \vartheta_{r}, \phi_{r}\right) \\
& \tan \left(\boldsymbol{\kappa}_{-1}^{\mathrm{tx}} / 2\right)\left(\boldsymbol{K}_{t}^{\mathrm{x}} \boldsymbol{T}_{t x} \otimes \boldsymbol{I}_{M_{r}}\right) \boldsymbol{h}\left(\vartheta_{t}, \phi_{t} ; \vartheta_{r}, \phi_{r}\right)=\left(\boldsymbol{K}_{t}^{x 2} \boldsymbol{T}_{t x} \otimes \boldsymbol{I}_{M_{r}}\right) \boldsymbol{h}\left(\vartheta_{t}, \phi_{t} ; \vartheta_{r}, \phi_{r}\right) \\
& \tan \left(\boldsymbol{\kappa}_{-1}^{r y} / 2\right)\left(\boldsymbol{K}_{r}^{y 1} \boldsymbol{T}_{r y} \otimes \boldsymbol{I}_{M_{t}}\right) \tilde{h}\left(\vartheta_{t}, \phi_{t} ; \vartheta_{r}, \phi_{r}\right)=\left(\boldsymbol{K}_{r}^{y 2} \boldsymbol{T}_{r y} \otimes \boldsymbol{I}_{M_{t}}\right) \tilde{h}\left(\vartheta_{t}, \phi_{t} ; \vartheta_{r}, \phi_{r}\right) \\
& \tan \left(\boldsymbol{\kappa}_{-1}^{r x} / 2\right)\left(\boldsymbol{K}_{r}^{x 1} \boldsymbol{T}_{r x} \otimes \boldsymbol{I}_{M_{t}}\right) \tilde{h}\left(\vartheta_{t}, \phi_{t} ; \vartheta_{r}, \phi_{r}\right)=\left(\boldsymbol{K}_{r}^{x 2} \boldsymbol{T}_{r x} \otimes \boldsymbol{I}_{M_{t}}\right) \tilde{h}\left(\vartheta_{t}, \phi_{t} ; \vartheta_{r}, \phi_{r}\right)
\end{aligned}
$$

Where

$$
\begin{aligned}
& \boldsymbol{T}_{t y}=\boldsymbol{Q}_{2 M_{M^{y}}-1}^{H} \boldsymbol{J}_{t y} \boldsymbol{Q}_{M_{t}} \quad \boldsymbol{T}_{r x}=\boldsymbol{Q}_{2 M_{r x}-1}^{H} \boldsymbol{J}_{r x} \boldsymbol{Q}_{M_{r}} \quad \boldsymbol{T}_{t x}=\boldsymbol{Q}_{2 M_{x}-1}^{H} \boldsymbol{J}_{t x} \boldsymbol{Q}_{M_{t}} \quad \boldsymbol{T}_{r y}=\boldsymbol{Q}_{2 M_{r y}-1}^{H} \boldsymbol{J}_{r y} \boldsymbol{Q}_{M_{r}} \\
& \boldsymbol{K}_{t}^{y 1}=\operatorname{Re}\left\{\boldsymbol{Q}_{2 M_{y y}-2}^{H} \boldsymbol{J}_{t}^{y 2} \boldsymbol{Q}_{2 M_{y y}-1}\right\} \quad \boldsymbol{K}_{t}^{y 2}=\operatorname{Im}\left\{\boldsymbol{Q}_{2 M_{y y}-2}^{H} \boldsymbol{J}_{t}^{y 2} \boldsymbol{Q}_{2 M_{t y}-1}\right\} \\
& \boldsymbol{K}_{t}^{x 1}=\operatorname{Re}\left\{\boldsymbol{Q}_{2 M_{t x}-2}^{H} \boldsymbol{J}_{t}^{\chi 2} \boldsymbol{Q}_{2 M_{t x}-1}\right\} \quad \boldsymbol{K}_{t}^{\chi 2}=\operatorname{Im}\left\{\boldsymbol{Q}_{2 M_{t x}-2}^{H} \boldsymbol{J}_{t}^{x 2} \boldsymbol{Q}_{2 M_{t x}-1}\right\} \\
& \boldsymbol{K}_{r}^{y 1}=\operatorname{Re}\left\{\boldsymbol{Q}_{2 M_{r y}-2}^{H} \boldsymbol{J}_{r}^{y 2} \boldsymbol{Q}_{2 M_{r y}-1}\right\} \quad \boldsymbol{K}_{r}^{y 2}=\operatorname{Im}\left\{\boldsymbol{Q}_{2 M_{r y}-2}^{H} \boldsymbol{J}_{r}^{y 2} \boldsymbol{Q}_{2 M_{r y}-1}\right\} \\
& \boldsymbol{K}_{r}^{\times 1}=\operatorname{Re}\left\{\boldsymbol{Q}_{2 M_{r x}-2}^{H} \boldsymbol{J}_{r}^{\times 2} \boldsymbol{Q}_{2 M_{r x}-1}\right\} \quad \boldsymbol{K}_{r}^{\times 2}=\operatorname{Im}\left\{\boldsymbol{Q}_{2 M_{r_{x}-2}}^{H} \boldsymbol{J}_{r}^{\times 2} \boldsymbol{Q}_{2 M_{r_{x}}-1}\right\}
\end{aligned}
$$

And $\tilde{\boldsymbol{h}}(\vartheta, \phi)=\boldsymbol{h}_{t}(\vartheta, \phi) \otimes \boldsymbol{h}_{r}(\vartheta, \phi)=\boldsymbol{\Xi}_{M_{t} M_{r}} \boldsymbol{h}(\vartheta, \phi), \boldsymbol{\Xi}_{M_{t} M_{r}}=\sum_{m=1}^{M_{r}} \sum_{n=1}^{M_{t}}\left(\boldsymbol{\Xi}_{m, n}^{M_{r} \times M_{t}} \otimes \boldsymbol{\Xi}_{n, m}^{M_{t} \times M_{r}}\right)$. Where $\Xi_{i, j}^{M_{r} \times M_{t}}$ is the $M_{r} \times M_{t}$ matrix with one on its ith row and $j$ th column and zeros elsewhere. Taking into account all the $\mathrm{K}$ targets and the relationship between $\boldsymbol{U}_{s}$ and $\boldsymbol{H}$, we can get

$$
\begin{array}{ll}
\left(\boldsymbol{K}_{t}^{y 1} \boldsymbol{T}_{t y} \otimes \boldsymbol{I}_{M_{r}}\right) \boldsymbol{U}_{s} \boldsymbol{\Psi}_{t y}=\left(\boldsymbol{K}_{t}^{y 2} \boldsymbol{T}_{t y} \otimes \boldsymbol{I}_{M_{r}}\right) \boldsymbol{U}_{s} & \left(\boldsymbol{K}_{t}^{x 1} \boldsymbol{T}_{t x} \otimes \boldsymbol{I}_{M_{r}}\right) \boldsymbol{U}_{s} \boldsymbol{\Psi}_{t x}=\left(\boldsymbol{K}_{t}^{x 2} \boldsymbol{T}_{t x} \otimes \boldsymbol{I}_{M_{r}}\right) \boldsymbol{U}_{S} \\
\left(\boldsymbol{K}_{r}^{y 1} \boldsymbol{T}_{r y} \otimes \boldsymbol{I}_{M_{t}}\right) \tilde{\boldsymbol{U}}_{s} \boldsymbol{\Psi}_{r y}=\left(\boldsymbol{K}_{r}^{y 2} \boldsymbol{T}_{r y} \otimes \boldsymbol{I}_{M_{t}}\right) \tilde{\boldsymbol{U}}_{s} & \left(\boldsymbol{K}_{r}^{\chi 1} \boldsymbol{T}_{r x} \otimes \boldsymbol{I}_{M_{t}}\right) \tilde{\boldsymbol{U}}_{s} \boldsymbol{\Psi}_{r x}=\left(\boldsymbol{K}_{r}^{\chi 2} \boldsymbol{T}_{r x} \otimes \boldsymbol{I}_{M_{t}}\right) \tilde{\boldsymbol{U}}_{S}
\end{array}
$$

Where $\tilde{\boldsymbol{U}}_{s}=\boldsymbol{\Xi}_{M_{t} M_{r}} \boldsymbol{U}_{s} ; \boldsymbol{\Psi}_{t y}=\boldsymbol{T}^{-1} \boldsymbol{\Phi}_{t y} \boldsymbol{T}, \boldsymbol{\Psi}_{t x}=\boldsymbol{T}^{-1} \boldsymbol{\Phi}_{t x} \boldsymbol{T}, \boldsymbol{\Psi}_{r y}=\boldsymbol{T}^{-1} \boldsymbol{\Phi}_{r y} \boldsymbol{T}, \boldsymbol{\Psi}_{r x}=\boldsymbol{T}^{-1} \boldsymbol{\Phi}_{r x} \boldsymbol{T}$,

$$
\begin{array}{ll}
\boldsymbol{\Phi}_{t y}=\operatorname{diag}\left\{\tan \left(\kappa_{-1}^{t y, 1} / 2\right), \cdots, \tan \left(\kappa_{-1}^{t y, K} / 2\right)\right\} & \boldsymbol{\Phi}_{t x}=\operatorname{diag}\left\{\tan \left(\kappa_{-1}^{t x, 1} / 2\right), \cdots, \tan \left(\kappa_{-1}^{t x, K} / 2\right)\right\} \\
\boldsymbol{\Phi}_{r y}=\operatorname{diag}\left\{\tan \left(\kappa_{-1}^{r y, 1} / 2\right), \cdots, \tan \left(\kappa_{-1}^{r y, K} / 2\right)\right\} & \boldsymbol{\Phi}_{r x}=\operatorname{diag}\left\{\tan \left(\kappa_{-1}^{r x, 1} / 2\right), \cdots, \tan \left(\kappa_{-1}^{r x, K} / 2\right)\right\}
\end{array}
$$

Lastly, we can obtain the corresponding 2D-DOA and 2D-DOD estimation via(14).

$$
\begin{aligned}
& \hat{u}_{t y}^{k}=2 \operatorname{atan}\left(\tilde{\boldsymbol{\Phi}}_{t y}^{k}\right) / \pi \quad \hat{v}_{t x}^{k}=2 \operatorname{atan}\left(\tilde{\boldsymbol{\Phi}}_{t x}^{k}\right) / \pi \quad \hat{u}_{r y}^{k}=2 \operatorname{atan}\left(\tilde{\boldsymbol{\Phi}}_{r y}^{k}\right) / \pi \quad \hat{v}_{r x}^{k}=2 \operatorname{atan}\left(\tilde{\boldsymbol{\Phi}}_{r x}\right) / \pi \\
& \widehat{\phi}_{t k}=\arccos \left(\sqrt{\left(\hat{u}_{t y}^{k}\right)^{2}+\left(\hat{v}_{t x}^{k}\right)^{2}}\right) \quad \widehat{\vartheta}_{t k}=\arctan \left(\hat{u}_{t y}^{k} / \hat{v}_{t x}^{k}\right) \\
& \widehat{\phi}_{r k}=\arccos \left(\sqrt{\left(\hat{u}_{r y}^{k}\right)^{2}+\left(\hat{v}_{r x}^{k}\right)^{2}}\right) \quad \widehat{\vartheta}_{r k}=\arctan \left(\hat{u}_{r y}^{k} / \widehat{v}_{r x}^{k}\right)
\end{aligned}
$$

Where $\tilde{\boldsymbol{\Phi}}_{t y}^{k}, \tilde{\boldsymbol{\Phi}}_{t x}^{k}, \tilde{\boldsymbol{\Phi}}_{r y}^{k}$ and $\tilde{\boldsymbol{\Phi}}_{r x}^{k}$ corresponding the $k$ th $(k=1, \cdots, K)$ eigenvalue of eigenmatrix respectively. 


\section{Simulation Results}

In this section, Monte Carlo simulations are presented to assess the angle estimation performance of the algorithms. Assume that there exist three targets located at $\left(\vartheta_{t 1}, \phi_{t 1}, \vartheta_{r 1}, \phi_{r 1}\right)=\left(10^{\circ}, 15^{\circ}, 20^{\circ}, 25^{\circ}\right)$, $\left(\vartheta_{t 2}, \phi_{t 2}, \vartheta_{r 2}, \phi_{r 2}\right)=\left(20^{\circ}, 25^{\circ}, 30^{\circ}, 35^{\circ}\right),\left(\vartheta_{t 3}, \phi_{t 3}, \vartheta_{r 3}, \phi_{r 3}\right)=\left(30^{\circ}, 35^{\circ}, 40^{\circ}, 45^{\circ}\right)$. MIMO radar equipped with $M_{t x}=3, M_{t x}=3, M_{r x}=3, M_{r y}=3, M_{t}=M_{r}=9$; Snapshot number is 200 .

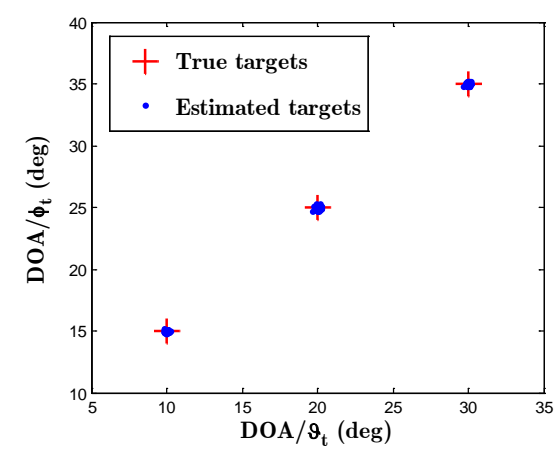

(a) The estimation results of the 2D-DOA

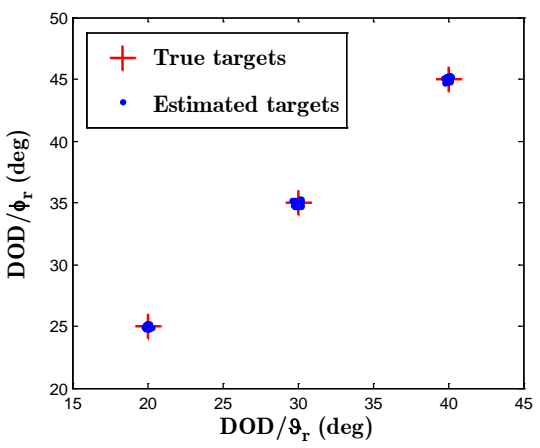

(b) The estimation results of 2D-DOD

Figure.2 The estimation results of proposed algorithm

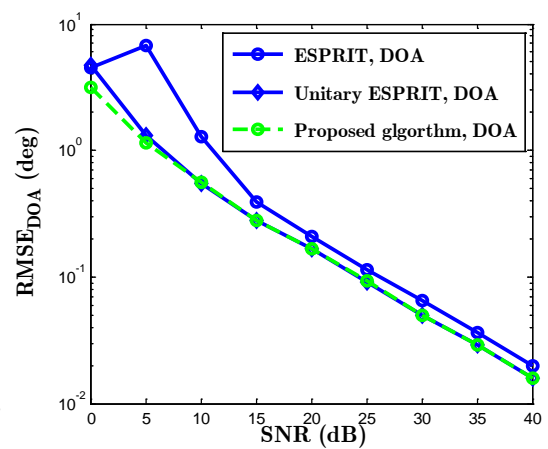

(a) Performance of the 2D-DOA versus SNR

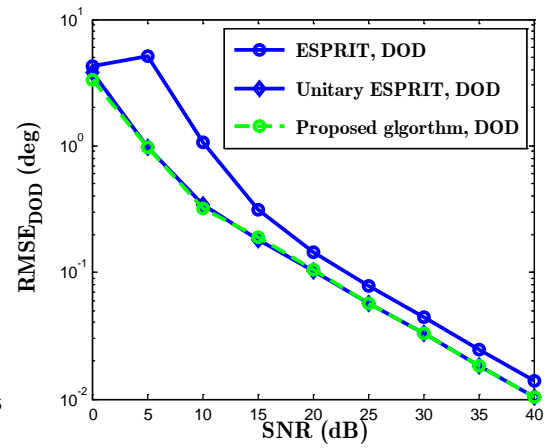

(b) Performance of the 2D-DOD versus SNR

Figure.3 Angles estimation performance comparison with different methods versus SNR

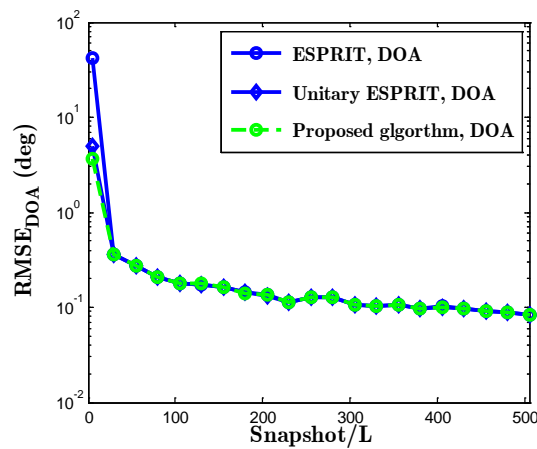

(a) Performance of the 2D-DOA versus snapshot

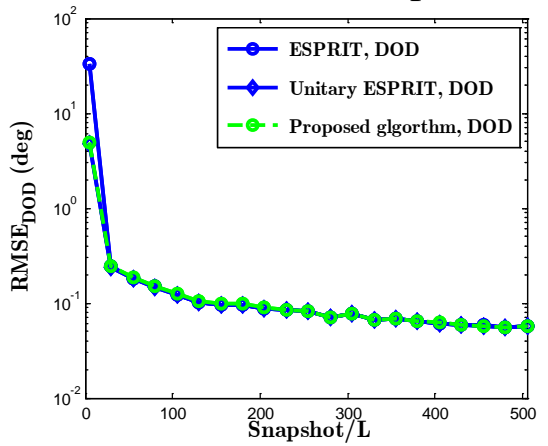

(b) Performance of the 2D-DOD versus snapshot Figure.4 Angles estimation performance comparison with different methods versus snapshot

Figure 2 depicts the paired results of three targets from 200 Monte Carlo runs with $\mathrm{SNR}=10 \mathrm{~dB}$, which shows that the 2D-DOA and 2D-DOD are paired correctly in our scheme. Cross and dot points represent the true location of target and estimation result, respectively. To further demonstrate the statistical efficiency of the proposed method, we compare the estimation accuracy of the proposed method with the ESPRIT and Unitary ESPRIT algorithm in[7,8].Assume that receive and transmit array satisfy $M_{t x}=4, M_{t x}=4, M_{r x}=4, M_{r y}=4, M_{t}=M_{r}=13$. and the other simulation conditions are the same as the above simulation ones. Figures 3 shows the RMSE of 2D-DOA and 2D-DOD for different methods versus SNR with snapshot length is 200 , respectively. And Figures 4 shows the RMSE of 2D-DOA and 2D-DOD for different methods versus snapshot with $\mathrm{SNR}=10 \mathrm{~dB}$, respectively. Therefore, it is seen that the proposed method has the better estimation performance.

\section{Conclusion}

In this paper, the issue of two-dimensional (2D) direction of arrival (DOA) and two-dimensional (2D) direction of departure (DOD) estimation for bistatic multiple-input-multiple-output (MIMO) radar with cross array is investigated. A novel multidimensional angle estimation methods based on unitary transformation is proposed. The proposed method, without costing the aperture of array and peak searching, can realize multidimensional angle estimation with automatic pairing and provides 
better estimation performance than ESPRIT. Compared with the traditional unitary transformation, the steering vectors of transmit and receive array can be turned into a real-value field via the unitary matrix and transformation in our scheme, respectively, Therefore, the proposed method can realize that the eigen-value decomposition and parameter estimation can be computed absolutely in a real-value field, with a result of a lower computation complexity.

\section{Acknowledgement}

In this paper, the research is supported by national science foundation of China (60702015), Auhui province foundation for science and technology research project (1310115188), scientific research foundation of Electronic Engineering Institute (KY13A197, KY13A200, KY13A206).

\section{References}

[1] Fisher E, Haimovich A, Blum R S, et al. Spatial Diversity in Radar Models and Detection Performance [J]. IEEE Transactions on Signal Processing, 2006, 54(3): 823-838.

[2] Li J, Stoica P, Xu L, Roberts W. On parameter identifiability of MIMO radar [J]. IEEE Signal Processing Letters, 2007, 14(12): 968-971.

[3] $\mathrm{Xu} \mathrm{L,} \mathrm{Li} \mathrm{J,} \mathrm{Stoica} \mathrm{P.} \mathrm{Target} \mathrm{detection} \mathrm{and} \mathrm{parameter} \mathrm{estimation} \mathrm{for} \mathrm{MIMO} \mathrm{radar}$ systems[J].IEEE Transactions on Aerospace and Electronic Systems, 2008,44(3): 927-939.

[4] Bekkerman I, Tabrikian J. Target detection and localization using MIMO radars and sonars[J].IEEE Transactions on Signal Processing, 2006,5(10):3873-3883.

[5] Tang B, Tang J, Zhang Y, Zheng Z D. Maximum Likelihood Estimation of DOD and DOA for Bistatic MIMO Radar [J]. Signal Processing, 2013,93(5): 1349-1357.

[6] M. L. Bencheikh and Y. Wang, Joint DOD-DOA Estimation using Combined ESPRIT-MUSIC Approach in MIMO Radar [J]. IEE Electronics Letters, 2010, 46(15): 2686-2691.

[7] Chen J, Gu H, Su W. Angle estimation using ESPRIT without pairing in MIMO radar[J].Electronics Letters, 2008,44, 1422-1423.

[8] Zheng G, Chen B, Yang M. Unitary ESPRIT algorithm for bistatic MIMO radar [J].Electronics Letters,2012, 48, (3):179-181

[9] C. Yunhe, Joint estimation of angle and Doppler frequency for bistatic MIMO radar [J]. IEE Electronics Letters, 2010,46(2), 170-172.

[10] M.D. Zoltowski, M. Haardt, C.P. Mathews, Closed-form 2-D angle estimation with rectangular arrays in element space or beamspace via unitary ESPRIT [J]. IEEE Transactions on Signal Processing, 1996, 44(2), 316-328. 